WAFR'00

\title{
Positioning Symmetric and Non-Symmetric Parts using Radial and Constant Force Fields
}

\author{
Florent Lamiraux LAAS-CNRS, 31077 Toulouse, France \\ Lydia E. Kavraki Rice University MS-132, Houston, TX 77005, USA
}

Part positioning is an important task in manufacturing. New approaches have been proposed to perform this task using force fields implemented on an active surface. A part placed on such a surface is subjected to a resultant force and torque and moves toward a stable equilibrium configuration. Such force fields can be implemented using MEMS. In this paper, we study the combination of a unit radial field with a small constant force field. In prior work we proved that such a combination can uniquely position a class of non-symmetric parts. In this paper, we propose a more complete modeling of this combination which allows us to devise a method to determine all the equilibrium configurations of a part in the above force fields. This method works for both symmetric and non-symmetric parts. Beyond the method, this paper reports a comprehensive study of the action of radial and constant potential fields over parts with an original characterization of local minima of the lifted potential field.

\section{Introduction}

During manufacturing, parts typically stored in boxes have to be manipulated and oriented before assembly. This task is critical in manufacturing since it strongly affects the productivity of the assembly line. Orientation has been traditionally performed by vibratory bowl feeders. However, these devices are designed for a given part and need to be modified if the shape of the part changes.

Recent work has investigated alternative ways of orienting parts, and emphasis has been placed on simple programmable devices $[1,6,9,15]$. Part positioning without sensing has become very popular over the past few years since it is easy to implement and the methods proposed can be very robust $[1,3,6,7,8,9,16]$. Our work brings new contributions in this domain.

One of the pioneering papers in this area, [9], proposed to orient a polygonal part by a sequence of squeezes performed by two parallel jaw grippers. Given a polygonal part, the paper described an algorithm to compute the best sequence of squeezes that uniquely orients the convex hull of the part no matter what the initial configuration is. In [16], the parts are on a conveyor belt and rotate by contact with passive fences.

Another approach is based on force fields implemented in an horizontal plane [6, 13]. A part lying on the field is subjected to a resultant force and torque that move the part toward a stable equilibrium configuration if one exists. A series of papers (see [4] for detailed references) established the fundamentals for part manipulation using force fields. Current technology permits the implementation of certain force fields in the microscale with MEMS actuators [6] or air jets [2] and in the macroscale using mechanical devices [14]. Vibrating plates can also be used to produce certain force fields in the plane [3].

In work which is summarized in $[4,6]$ the properties of force fields that are suitable for sensorless manipulation were analyzed and manipulation strategies were proposed. Several fields were investigated including the squeeze, radial, and inertial fields and combinations thereof. Notably the algorithm of [9] still applies when the jaw grippers are replaced by squeeze force fields. Later work [10] introduced the elliptic potential field which gave rise to two stable equilibria for non symmetric parts. 


\section{Complexity and Uniqueness of Positioning}

There are two main issues when positioning ${ }^{1}$ parts using force fields. The first one is the number of basic operations, (i.e. , number of force fields used) and the second one is the number of distinct positions that the strategy yields. Both issues have been addressed previously and work has been done both on finding shorter sequences of operations [6] and on finding a unique operation that positions a part. In [10], a quadratic potential field is used to position parts. Any part with distinct principal moments of inertia has exactly two stable equilibrium configurations in these fields. The other parts have undefined orientation. Addressing the above issues at the same time would consist in finding a single field that uniquely positions a part. It was conjectured in [6] that the combination of a unit radial field with a constant force field would fulfill this task. In [5], we proved that this conjecture is true under the condition that when the part is subjected to the unit radial field only, the center of mass of the part in equilibrium is not at the origin of the field. Thus, for almost any non-symmetric part, there exists a force field that uniquely positions this part.

\section{Contributions and Organization of the Paper}

Symmetric parts like a rectangle or regular polygons for instance do not fulfill the above condition: when placed in a unit radial field, at equilibrium, the center of mass of the part will coincide with the origin of the unit radial field, due to symmetry. The goal of this paper is to determine all the stable equilibrium configurations of a general part, symmetric or not, subjected to the combination of a unit radial field with a small constant field. There are two main contributions in this paper. The first one is a characterization of the stable equilibrium configurations of a part using a general modeling based on the curve of translational equilibria for fixed orientations and its partial derivatives. This modeling is a generalization of results previously published $[5,12]$. The second contribution is a method to compute the above mentioned partial derivatives and an algorithm

\footnotetext{
${ }^{1}$ Orienting a part consists in specifying the orientation of the part. Positioning a part consists in specifying the position and orientation.
}

that determines all the equilibrium configurations of a given part.

This paper is organized as follows. In Section 2, we give some notation and definitions. In Section 3, we first define the unit radial field that we then combine with a constant field. We define the equilibrium curve as the locus of the translational equilibria for fixed orientation. After pointing out the smoothness of this curve, we state the central result of the paper that expresses the stable equilibrium configurations of a part w.r.t. the equilibrium curve and its partial derivatives. Section 4 first describes the practical computations of these partial derivatives. An algorithm to determine all the stable equilibrium configurations of a part is then given. Finally, an example of positioning of a symmetric part is presented. We conclude in Section 5.

\section{Notation and Definitions}

This section puts forward the notation and definitions used in the paper. We first define the action of a force field over a part and the notion of a stable equilibrium configuration in this context. Then we outline some properties of force fields deriving from a potential function. Most of these definitions have been introduced in [4].

If $E$ is a set, we denote by $\operatorname{int}(E)$ and $\partial E$ the interior and boundary of $E$. Throughout the paper, $\mathbf{r}$ denotes a point of the plane of coordinates $(\xi, \eta)$ in a frame attached to the plane. We consider a part in the plane occupying surface $S$ with center of mass $G$ at $(0,0)$ in a reference configuration $\mathbf{q}_{0}$. We suppose that $S$ is a compact set and that its boundary $\partial S$ is a zeromeasure subset of the plane. The configuration space of the part is $\mathcal{C}=\mathbf{R}^{2} \times \mathbf{S}^{1}$, where $\mathbf{S}^{1}$ is the unit circle. A configuration $\mathbf{q}=(x, y, \theta) \in \mathcal{C}$ corresponds to a rigidbody transformation $\varphi_{\mathbf{q}}$ in the plane transforming $\mathbf{r}=$ $(\xi, \eta)$ into

$$
\varphi_{\mathbf{q}}(\mathbf{r})=\left(\begin{array}{c}
x+\xi \cos \theta-\eta \sin \theta \\
y+\xi \sin \theta+\eta \cos \theta
\end{array}\right)
$$

We denote by $S_{\mathbf{q}}$ the subset occupied by the part in configuration $\mathbf{q}$.

When the part is placed on a force field $\mathbf{f}(\mathbf{r})$, the resultant force and torque it is subjected to are the 
following:

$$
\begin{aligned}
& \mathbf{F}(\mathbf{q})=\int_{S \mathrm{q}} \mathbf{f}(\mathbf{r}) d \mathbf{r} \\
& M(\mathbf{q})=\int_{S \mathrm{q}}\left(\mathbf{r}-\mathbf{r}_{G}\right) \times \mathbf{f}(\mathbf{r}) d \mathbf{r}
\end{aligned}
$$

where $\mathbf{r}_{G}=(x, y)$ is the position of the center of mass $G$, when the part is at configuration $\mathbf{q}$.

We say that $\mathbf{q}$ is an equilibrium configuration if the resultant force and torque vanish at $\mathbf{q}$. Moreover, an equilibrium configuration $\mathbf{q}$ is said to be stable if, subjected to a small perturbation, the part stays in the neighborhood of $\mathbf{q}$.

Potential Fields The class of force fields deriving from a potential function are very helpful in mechanics and electrostatics since the equilibrium configurations of a point-particle subjected to such a field are exactly the local minima of the potential field. This property extends naturally for "reasonable" force fields from the plane to the configuration space via the notion of lifted potential field [4].

\section{Definition 1 (Lifted Potential Field)}

Let $\mathbf{f}(\mathbf{r})$ be a force field in the plane deriving from a potential function $u$, i.e., $\mathbf{f}(\mathbf{r})=-\nabla u(\mathbf{r})$. The function

$$
U(\mathbf{q})=\int_{S \mathrm{q}} u(\mathbf{r}) d \mathbf{r}=\int_{S} u\left(\varphi_{\mathbf{q}}(\mathbf{r})\right) d \mathbf{r}
$$

over $\mathcal{C}$ is called the lifted potential field induced by $u$.

The lifted potential field $U(\mathbf{q})$ is thus defined by integrating the value of the plane potential field over the surface $S_{\mathbf{q}}$ occupied by the part in configuration $\mathbf{q}$. In [6] some properties of the lifted potential field are established. The most interesting among these properties is that the partial derivatives of the lifted potential field w.r.t. $x, y$ and $\theta$ are exactly the opposite of respectively the coordinates of the resultant force and the resultant torque.

$$
\begin{aligned}
& \frac{\partial U}{\partial x}(\mathbf{q})=-F_{x}(\mathbf{q}) \\
& \frac{\partial U}{\partial y}(\mathbf{q})=-F_{y}(\mathbf{q})
\end{aligned}
$$

$$
\frac{\partial U}{\partial \theta}(\mathbf{q})=-M(\mathbf{q})
$$

The main consequence of these equalities is that stable equilibrium configurations are equivalent to local minima of the lifted potential. For this reason, we focus our attention in the following sections on the local minima of the lifted potential field induced by radial and constant fields.

\section{Radial and Constant Fields}

In this section we study the properties of combinations of a unit radial force field with a small constant force field. Both fields derive from a potential. We give a method to determine the stable equilibrium configurations (i.e. , local minima) of a part subjected to such a combination in the general case. See also [5], were some of the material below was originally developed.

\subsection{Unit Radial Field}

We call unit radial field the force field $\mathbf{f}(\mathbf{r})=-\frac{\mathbf{r}}{\|\mathbf{r}\|}$. This field has constant magnitude, is oriented toward the origin of the frame attached to the plane and derives from the potential function

$$
v(\mathbf{r})=\|\mathbf{r}\|=\sqrt{\xi^{2}+\eta^{2}}
$$

$v$ is clearly symmetric by rotation about the origin. Thus if $\mathbf{q}^{\prime}$ is obtained from $\mathbf{q}$ by a rotation about the origin, the values of the lifted potential field at $\mathbf{q}$ and $\mathbf{q}^{\prime}$ are the same. To take fully advantage of this property, we are going to use a new system of coordinates $(X, Y, \theta)$ for the configuration of the part, illustrated in Figure 1 and defined by:

$$
\begin{aligned}
& X=x \cos \theta+y \sin \theta \\
& Y=-x \sin \theta+y \cos \theta
\end{aligned}
$$

Expressed in this system of coordinates, the lifted potential field $V$ corresponding to $v$ depends only on $X$ and $Y$ and can be written as follows:

$$
V(X, Y, \theta)=\int_{S} v(X+\xi, Y+\eta) d \xi d \eta
$$

Because of the independence on $\theta$, we will consider $V$ as a function of $(X, Y)$ only. Notice that when $\theta=0$, 


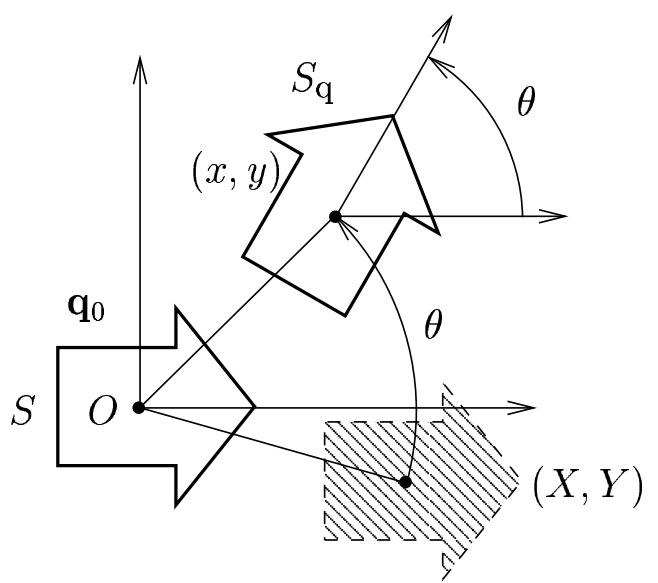

Figure 1: Parameterization of $\mathcal{C}$ with the system of coordinates $\mathbf{q}=(X, Y, \theta) . \varphi \mathbf{q}$ corresponds to a translation of $(X, Y)$ followed by a rotation of angle $\theta$ about the origin.

$X=x$ and $Y=y \cdot V(X, Y)=V(x, y, 0)$ can thus be considered as the lifted potential field of the part in translation. This interpretation can be helpful to understand some of the forthcoming developments.

Smoothness of $V$ In the following sections, we will use a lot the partial derivatives of $V$. For this reason, we state in this section the differentiability properties of this function.

$\mathcal{C}$ is partitioned into three subsets. The configurations for which the origin of the radial field is in the interior of the part, the configuration for which the origin is outside the part and the configurations for which the origin is on the boundary of the part. We denote respectively $\mathcal{C}^{\text {in }}, \mathcal{C}^{\text {out }}$ and $\mathcal{C}^{\text {bound }}$ these subsets. To decide in which of these subsets a configuration $(X, Y, \theta)$ lies, we need only to consider $(X, Y)$ in the new system of coordinates. (see Figure 3.1 for intuition):

$$
\begin{array}{lll}
\mathbf{q} \in \mathcal{C}^{\text {in }} & \Leftrightarrow & (-X,-Y) \in \operatorname{int}(S) \\
\mathbf{q} \in \mathcal{C}^{\text {out }} & \Leftrightarrow & (-X,-Y) \notin S \\
\mathbf{q} \in \mathcal{C}^{\text {bound }} & \Leftrightarrow & (-X,-Y) \in \partial S
\end{array}
$$

As far as differentiability is concerned, $V$ has the following properties.

Proposition 2 (i) $V$ is of class $C^{\infty}$ over $\mathcal{C}^{\text {in }}$ and over $\mathcal{C}^{\text {out }}$, (ii) $V$ is of class $C^{2}$ over $\mathcal{C}$ and

$$
\begin{aligned}
\frac{\partial V}{\partial X}(X, Y) & =\int_{\mathrm{S}} \frac{\partial v}{\partial \xi}(X+\xi, Y+\eta) d \xi d \eta \\
\frac{\partial V}{\partial Y}(X, Y) & =\int_{\mathrm{S}} \frac{\partial v}{\partial \eta}(X+\xi, Y+\eta) d \xi d \eta \\
\frac{\partial^{2} V}{\partial X^{2}}(X, Y) & =\int_{\mathrm{S}} \frac{\partial^{2} v}{\partial \xi^{2}}(X+\xi, Y+\eta) d \xi d \eta \\
\frac{\partial^{2} V}{\partial Y^{2}}(X, Y) & =\int_{\mathrm{S}} \frac{\partial^{2} v}{\partial \eta^{2}}(X+\xi, Y+\eta) d \xi d \eta \\
\frac{\partial^{2} V}{\partial X \partial Y}(X, Y) & =\int_{\mathrm{S}} \frac{\partial^{2} v}{\partial \xi \partial \eta}(X+\xi, Y+\eta) d \xi d \eta
\end{aligned}
$$

The classical results of integration theory, regarding differentiating inside the integral cannot be applied as such to the unit radial field because of the non differentiability at the origin. The proof of this proposition is based on a family $v_{h}$ of approximations of $v, C^{\infty} \mathrm{ev}-$ erywhere and equal to the radial field outside the disc of radius $h$ centered at the origin. We do not give here the proof of this Proposition. This proof can be found in [11].

It is interesting to notice that the lifted potential field $V$ is $C^{\infty}$ although $v$ is not even differentiable. The singularity of $v$ at the origin affects the smoothness of $V$ only where the boundary of the part passes above this singularity.

Minimum of $V$ and Pivot Point Due to symmetry, the unit radial field obviously cannot uniquely position a part. However, for a fixed value of $\theta$, any part in translation has a unique stable equilibrium. This property is a consequence of the Proposition refprop:posdef below. This proposition was also given in [5]. It is repeated here for completeness. Notice that it provides a different proof for the existence and uniqueness of the pivot point defined below than the one given in [4].

Proposition $3 V$ verifies the following properties:

(i) The Hessian of $V$ Hess $V(X, Y)$ is positive definite everywhere in $\mathbf{R}^{2}$,

(ii) $V$ has a unique local minimum over $\mathbf{R}^{2}$. 
Proof: As Hess $V$ is a 2 by 2 matrix, (i) is equivalent to

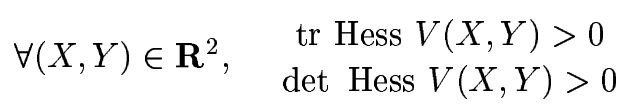

where tr and det are respectively the trace and determinant operators. According to (7-9) and (3), the second order partial derivatives of $V$ are

$$
\begin{aligned}
\frac{\partial^{2} V}{\partial X^{2}}(X, Y) & =\int_{\mathrm{S}} \frac{(Y+\eta)^{2}}{\left((X+\xi)^{2}+(Y+\eta)^{2}\right)^{3=}} d \xi d \eta \\
\frac{\partial^{2} V}{\partial Y^{2}}(X, Y) & =\int_{\mathrm{S}} \frac{(X+\xi)^{2}}{\left((X+\xi)^{2}+(Y+\eta)^{2}\right)^{3}=} d \xi d \eta \\
\frac{\partial V}{\partial X \partial Y}(X, Y) & =\int_{\mathrm{S}} \frac{-(X+\xi)(Y+\eta)}{\left((X+\xi)^{2}+(Y+\eta)^{2}\right)^{3}=2} d \xi d \eta
\end{aligned}
$$

It is straightforward from these expressions that $\operatorname{tr}$ Hess $V=\frac{\partial^{2} V}{\partial X^{2}}+\frac{\partial^{2} V}{\partial Y^{2}}$ is positive everywhere. The determinant of the Hessian of $V$

$$
\operatorname{det} \operatorname{Hess} V(X, Y)=\left(\frac{\partial^{2} V}{\partial X^{2}} \frac{\partial^{2} V}{\partial Y^{2}}-\left(\frac{\partial^{2} V}{\partial X \partial Y}\right)^{2}\right)(X, Y)
$$

is the sum of two terms, each of which is a product of two integrals over $S$. Replacing these products by integrals over the Cartesian product $S^{2}=S \times S$, we get

$$
\begin{aligned}
& \left(\int_{\mathrm{S}} f(\xi, \eta) d \xi d \eta\right)\left(\int_{\mathrm{S}} g(\xi, \eta) d \xi d \eta\right)= \\
& \int_{\mathrm{S}_{2}} f\left(\xi_{1}, \eta_{1}\right) g\left(\xi_{2}, \eta_{2}\right) d \xi_{1} d \eta_{1} d \xi_{2} d \eta_{2}
\end{aligned}
$$

If we condense the notation as follows, $X_{i}=\left(X+\xi_{i}\right)$, $Y_{i}=\left(Y+\eta_{i}\right)$ for $i=1,2$, and omit $d \xi_{1} d \eta_{1} d \xi_{2} d \eta_{2}$, we have

$$
\begin{aligned}
\operatorname{det} \operatorname{Hess} V(X, Y) & =\int_{\mathrm{S}_{2}} \frac{X_{1}^{2} Y_{2}^{2}}{\left(X_{1}^{2}+Y_{1}^{2}\right)^{3=}\left(X_{2}^{2}+Y_{2}^{2}\right)^{3=2}} \\
& -\int_{\mathrm{S}_{2}} \frac{X_{1} Y_{1} X_{2} Y_{2}}{\left(X_{1}^{2}+Y_{1}^{2}\right)^{3=}\left(X_{2}^{2}+Y_{2}^{2}\right)^{3=}} \\
& =\int_{\mathrm{S}_{2}} \frac{Y_{1}^{2} X_{2}^{2}-X_{1} Y_{1} X_{2} Y_{2}}{\left(X_{1}^{2}+Y_{1}^{2}\right)^{3=2}\left(X_{2}^{2}+Y_{2}^{2}\right)^{3=2}}
\end{aligned}
$$

In the first integral, $\left(X_{1}, Y_{1}\right)$ and $\left(X_{2}, Y_{2}\right)$ have a symmetric role and can be switched so that $X_{1}^{2} Y_{2}^{2}$ can be replaced by $\frac{1}{2}\left(X_{1}^{2} Y_{2}^{2}+X_{2}^{2} Y_{1}^{2}\right)$ and

$$
\begin{aligned}
\operatorname{det} \text { Hess } V(X, Y) & =\frac{1}{2} \int_{\mathrm{S}^{2}} \frac{X_{1}^{2} Y_{2}^{2}+X_{2}^{2} Y_{1}^{2}-2 X_{1} Y_{1} X_{2} Y_{2}}{\left(X_{1}^{2}+Y_{1}^{2}\right)^{3=2}\left(X_{2}^{2}+Y_{2}^{2}\right)^{3=2}} \\
& =\frac{1}{2} \int_{\mathrm{S}^{2}} \frac{\left(X_{1} Y_{2}-X_{2} Y_{1}\right)^{2}}{\left(X_{1}^{2}+Y_{1}^{2}\right)^{3=2}\left(X_{2}^{2}+Y_{2}^{2}\right)^{3}=2} \\
& >0 .
\end{aligned}
$$

Thus Hess $V$ is positive definite everywhere. This ensures us that if $V$ has a local minimum, it is unique.

Moreover, as $v(\mathbf{r})$ tends toward infinity when $\|\mathbf{r}\|$ tends toward infinity, $V(X, Y)$ also tends toward infinity as $(X, Y)$ diverges. This property implies the existence of a local minimum of $V$.

We denote by $\left(X_{0}, Y_{0}\right)$ the unique minimum of $V$. The set of equilibrium configurations of the part under the radial field is the following $\left\{\left(X_{0}, Y_{0}, \theta\right), \theta \in \mathbf{S}^{1}\right\}$. Let us express this curve in the standard system of coordinates:

$$
\begin{aligned}
& x=X_{0} \cos \theta-Y_{0} \sin \theta \\
& y=X_{0} \sin \theta+Y_{0} \cos \theta
\end{aligned}
$$

The stable equilibrium configurations are obtained by rotation of the part about the origin of the radial field. The point of the part situated at the origin in these configurations is called the pivot point and denoted $P[6]$. In the stable equilibrium configuration corresponding to $\theta=0$ (remember that in this case, $X=x, Y=y$.), the center of mass is translated to $\left(X_{0}, Y_{0}\right)$. Thus in configuration $\mathbf{q}_{0}$, the pivot point is at $\left(-X_{0},-Y_{0}\right)$ (see Figure 2).

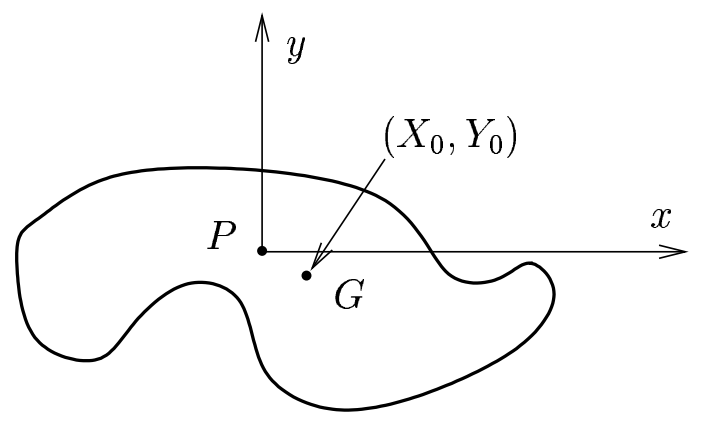

Figure 2: In a unit radial field, at the equilibrium configuration corresponding to $\theta=0$, the center of mass is at $\left(X_{0}, Y_{0}\right)$.

\subsection{Radial-Constant Field}

The previous section established the existence and uniqueness of the pivot point for a part subjected to the unit radial field. In this section, we perturb the radial field by adding a small constant field in order to 


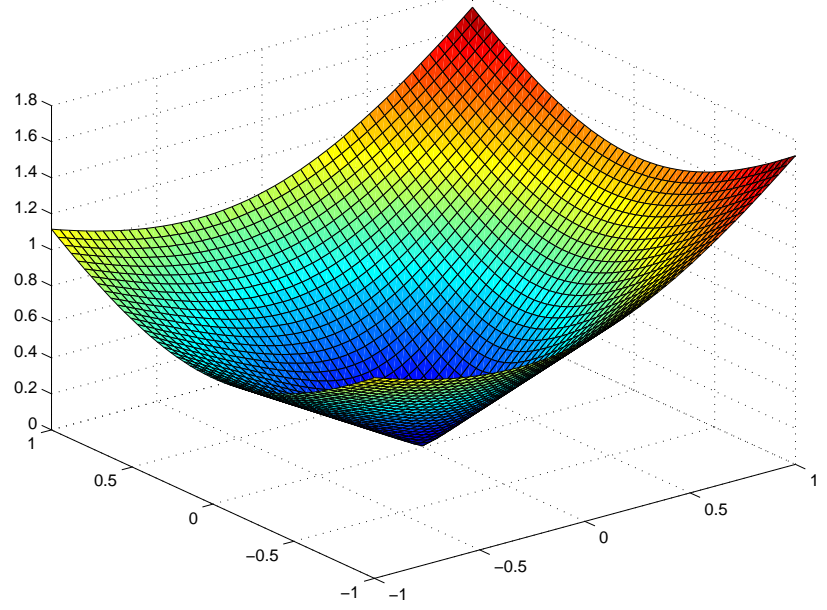

Figure 3: Potential function of the combination of a unit radial force field with a constant force field

break the symmetry. We are going to show that for each fixed orientation $\theta$ of the part, the corresponding lifted potential field has a unique minimum in $(X, Y)$. When now $\theta$ varies, the curve of these minima is $C^{1}$ and $C^{\infty}$ if the pivot point is not on the boundary of the part. We call this curve the equilibrium curve. Then we will give a characterization of the local minima of the lifted potential using the equilibrium curve.

We consider now the following potential function in the plane (Figure 3):

$$
u(\mathbf{r})=v(\mathbf{r})+\delta \eta
$$

where $v$ is the unit radial field and $\delta$ is a positive constant. The second term $\delta \eta$ corresponds to the constant force field $-\delta(0,1)$. The lifted potential field for a given value of $\delta$ is expressed as follows in the $(X, Y, \theta)$ system of coordinates:

$$
U_{\delta}(X, Y, \theta)=V(X, Y)+\delta|S|(\sin \theta X+\cos \theta Y)
$$

where $|S|$ is the area of $S$. For clarity purposes, we define the following functions

$$
U_{\theta, \delta}(X, Y)=U(X, Y, \theta, \delta)=U_{\delta}(X, Y, \theta)
$$

where the variables we put in the subscript are considered constant.
Minimum of $U_{\delta, \theta}$ and Equilibrium Curve The second term of the right hand side of Expression (12) is linear in $(X, Y)$. For this reason, $U_{\theta, \delta}$ has the same second order partial derivatives as $V$ and Hess $U_{\theta, \delta}=$ Hess $V$ is positive definite (Proposition 3). For any fixed value of $\theta$ and $\delta<1, u(\mathbf{r})$ tends toward infinity with $\|\mathbf{r}\|$. Thus $U_{\theta, \delta}(X, Y)$ tends toward infinity with $(X, Y)$ and $U_{\theta, \delta}$ has a unique local minimum. We denote by $\left(X^{*}(\theta, \delta), Y^{*}(\theta, \delta)\right)$ this local minimum. We can express it in the standard system of coordinates by:

$$
\begin{aligned}
& x^{*}(\theta, \delta)=\cos \theta X^{*}(\theta, \delta)-\sin \theta Y^{*}(\theta, \delta) \\
& y^{*}(\theta, \delta)=\sin \theta X^{*}(\theta, \delta)+\cos \theta Y^{*}(\theta, \delta)
\end{aligned}
$$

For each value of $\delta<1$, these local minima define a curve of parameter $\theta$ that we call equilibrium curve. We are going to show now that this curve is of class $C^{1}$. It is of class $C^{\infty}$ for small values of $\delta$ if the pivot point is not on the boundary of the part.

Notice that $\left(X^{*}, Y^{*}\right)(\theta, 0)=\left(X_{0}, Y_{0}\right)$ is the minimum of $V$ and therefore is independent of $\theta$. The smoothness of the equilibrium curve will prove useful in the sequel where its partial derivatives are used.

Proposition 4 The equilibrium curve is smooth.

(i) $X^{*}, Y^{*}, x^{*}$ and $y^{*}$ are continuously differentiable.

(ii) if $\left(-X_{0},-Y_{0}\right) \notin \partial S$ (i.e. , the pivot point is not on the boundary of the part), there exists $\delta_{0}$ such that $X^{*}, Y^{*}, x^{*}$, and $y^{*}$ are $C^{\infty}$ over $\mathbf{S}^{1} \times\left[0, \delta_{0}\right]$.

Proof: (i) This proposition is a direct result of the implicit function theorem. Indeed, if we define the following function from $\mathbf{R}^{4}$ into $\mathbf{R}^{2}$

$$
F:(X, Y, \theta, \delta) \rightarrow\left(\begin{array}{c}
\frac{\partial U}{\partial X}(X, Y, \theta, \delta) \\
\frac{\partial U}{\partial Y}(X, Y, \theta, \delta)
\end{array}\right)
$$

From (12), as $V$ is of class $C^{2}$ (Proposition 2), $F$ is of class $C^{1}$. By definition, the equilibrium curve minimizes the lifted potential field for fixed $\theta$ and $\delta$ and therefore fits the following implicit representation:

$$
F\left(X^{*}, Y^{*}, \theta, \delta\right)=0
$$


The differential of the partial function $F_{\theta, \delta}$ of the variables $(X, Y)$ is exactly the Hessian of $V$. From Proposition 3 , this differential is invertible everywhere. According to the implicit function theorem, these conditions imply that $X^{*}$ and $Y^{*}$ can be expressed as $C^{1}$ functions of $(\theta, \delta)$. As the equilibrium curve is unique, these $C^{1}$ functions are necessarily the formerly defined $X^{*}(\theta, \delta)$ and $Y^{*}(\theta, \delta)$.

(ii) If the pivot point is not on the boundary of the part, $-\left(X^{*}(\theta, 0), Y^{*}(\theta, 0)\right) \notin \partial S$. By the continuity of $X^{*}$ and $Y^{*}$, there exists a $\delta_{0}$ such that for any $\theta \in \mathbf{S}^{1}$ and $0 \leq \delta \leq \delta_{0},-\left(X^{*}(\theta, \delta), Y^{*}(\theta, \delta)\right) \notin \partial S$. In other words, if we follow the equilibrium curve for a small $\delta$, the origin of the field remains completely inside or completely outside the part and $\left(X^{*}, Y^{*}, \theta, \delta\right)$ remains in a domain where $F$ is smooth (from Proposition 2). Therefore, according to the implicit function theorem, $X^{*}$ and $Y^{*}$ are also smooth. Relations (13) and (14) imply that $x^{*}$ and $y^{*}$ have the same differentiability properties as $X^{*}$ and $Y^{*}$.

2

¿From now on, we will assume that the pivot point is not on the boundary of the part, so that the partial derivatives of the equilibrium curves are all defined for small $\delta$.

Properties of the equilibrium curve. We now point out a property of the equilibrium curves that will constitute the basis of our method to determine the local minima of $U$. For a fixed value of $\delta$ the local minima of $U$ are obviously on the equilibrium curve associated to $\delta$. We are going to show that these local minima are the points where $\left(x^{*}, y^{*}\right)$ crosses the $y$ axis from $x<0$ to $x>0$. For that, we define

$$
U_{\delta}^{*}(\theta)=U_{\delta}\left(X^{*}(\theta, \delta), Y^{*}(\theta, \delta), \theta\right)
$$

the minimum value of the lifted potential field for given $\theta$ and $\delta$. The variation of $U_{\delta}^{*}(\theta)$ along the equilibrium curve is given by the following proposition.

Proposition 5 For any $\theta \in \mathbf{S}^{1}$,

$$
\frac{d U_{\delta}^{*}}{d \theta}(\theta)=\delta|S| x^{*}(\theta, \delta)
$$

Proof: For clarity, we omit $\delta$ in the notation of this proof. By definition $U_{\delta}^{*}(\theta)=U_{\delta}\left(X^{*}(\theta), Y^{*}(\theta), \theta\right)$. Differentiating this expression w.r.t. to $\theta$ leads to

$$
\begin{aligned}
& \frac{d U \mathrm{ff}_{\mathrm{f}}}{d \theta}(\theta)=\frac{\partial U \mathrm{ffi}_{1}}{\partial X}\left(X \Lambda_{(\theta), Y} \Lambda_{(\theta), \theta)} \frac{d X^{\Lambda}}{d \theta}(\theta)+\right. \\
& \frac{\partial U_{\text {fff }}}{\partial Y}\left(X^{\Lambda_{(\theta), Y}} \Lambda_{(\theta), \theta)} \frac{d Y^{\Lambda}}{d \theta}(\theta)+\right. \\
& \frac{\partial U \mathrm{ffi}}{\partial \theta}\left(X^{\Lambda_{(\theta), Y}} \Lambda_{(\theta), \theta)}\right. \\
& =\frac{\partial U \mathrm{ffi}}{\partial \theta}\left(X^{\Lambda_{(\theta), Y}} \Lambda_{(\theta), \theta)}\right. \\
& =\delta|S|\left(\cos \theta X^{\Lambda}(\theta)-\sin \theta Y^{\Lambda}(\theta)\right) \\
& =\delta|S| x^{\Lambda}(\theta)
\end{aligned}
$$

using expression (12) and the fact that the partial derivatives of $U_{\delta}$ w.r.t. $X$ and $Y$ vanish at $\left(X^{*}, Y^{*}\right)$. 2

This proposition leads directly to the following property.

Proposition 6 For any fixed value of $\delta<1$, the two following properties are equivalent:

(i) $(X, Y, \theta)$ is a local minimum of $U_{\delta}$,

(ii) $X=X^{*}(\theta, \delta), Y=Y^{*}(\theta, \delta)$ and the equilibrium curve crosses the $y$-axis from left to right when $\theta$ jncreases: $x^{*}(\theta, \delta)=0$ and $\frac{\partial x}{\partial \theta}(\theta, \delta)>0$.

Figure 4 shows the value of the lifted potential along the equilibrium curve for a given part.

We are going now to devise a method for computing these equilibrium configurations for small values of $\delta$ and for any kind of part, symmetric or not.

\subsection{Computation of the Stable Equilibrium Configurations of a Part for Small $\delta$}

In [5], we proved that for a part with distinct pivot point and center of mass $\left(X_{0}, Y_{0}\right) \neq(0,0)$, the stable equilibrium configuration is unique. The proof is based on the expression (10) of the equilibrium curve of parameter $\delta=0$ and on the continuity of $x^{*}(\theta, \delta)$ and its partial derivatives. More precisely, $x^{*}(\theta, 0)$ vanishes for two values of $\theta: \theta_{1}$ and $\theta_{2}=\theta_{1}+\pi . x^{*}(\theta, 0)$ is increasing at one of these two values (say $\theta_{2}$ ) and decreasing at the other one.

$$
\begin{array}{ll}
x^{*}\left(\theta_{1}, 0\right)=0 & \frac{\partial x \Lambda}{\partial \theta}\left(\theta_{1}, 0\right)<0 \\
x^{*}\left(\theta_{2}, 0\right)=0 & \frac{\partial x}{\partial \theta}\left(\theta_{2}, 0\right)>0
\end{array}
$$




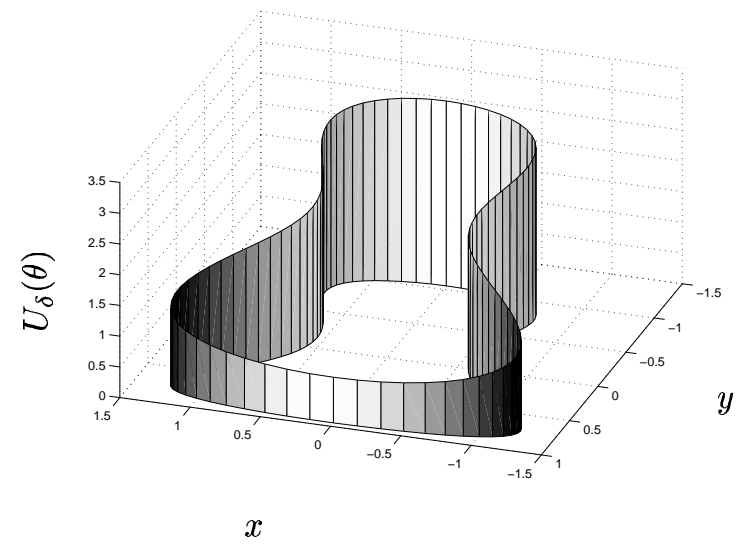

Figure 4: Along the equilibrium curve, the variation of the value of the lifted potential is proportional to $x^{\Lambda}$. Thus the stable equilibrium configurations of the part are those where the equilibrium curve crosses the $y$-axis from left to right.

By continuity of $x^{*}$ and $\frac{\partial x}{\partial \theta}$, we proved that for small values of $\delta, x^{*}(\theta, \delta)$ still vanishes only twice, around $\theta_{1}$ and $\theta_{2}$ and therefore the stable equilibrium configuration is unique.

When the pivot point and the center of mass are the same (that is when $\left.\left(X_{0}, Y_{0}\right)=(0,0)\right)$, this reasoning does not apply since from (10) and (13), $x^{*}(\theta, 0)=0$ for all $\theta$. The following theorem however, states that the criterion applied previously to $x^{*}(\theta, 0)$ can be applied to the first non-uniformly zero partial derivative of $x^{*}$ w.r.t. $\delta$, evaluated for $\delta=0: \frac{\partial^{n} x}{\partial \delta^{n}}(\theta, 0)$. More precisely, if $\frac{\partial^{n} x}{\partial \delta^{n}}(\theta, 0)$ has only simple roots, i.e. , vanishes with non zero slope then for small $\delta, x^{*}(\theta, \delta)$ also has only simple roots close to the roots of $\frac{\partial^{n} x}{\partial \delta^{n}}(\theta, 0)$ (Figure 5). Among those roots, some represents stable equilibrium configurations, the other ones are unstable.

Theorem 7 For a given part, if there exists an integer $n \geq 0$ such that

(i) for any $k$ such that $0 \leq k \leq n-1$, $\frac{\partial^{k} x^{\Lambda}}{\partial \delta^{k} \Lambda}(\theta, 0)=0$ uniformly over $\mathbf{S}^{1}$,

(ii) $\frac{\partial^{n} x \Lambda}{\partial \delta^{n}}(\theta, 0)$ vanishes only at a finite number of points $\left(\theta_{1}, \ldots, \theta_{2 m}\right)$, and

(iii) for any $1 \leq l \leq m, \frac{\partial^{n+1} x^{n}}{\partial \theta \partial \delta^{n}}\left(\theta_{2 l}, 0\right)>0$, $\frac{\partial^{n+1} x}{\partial \theta \partial \delta^{n}}\left(\theta_{2 l-1}, 0\right)<0$, then for small values of $\delta$, the part has exactly $m$ stable equilibrium configurations. These configurations converge toward $\left(X_{0}, Y_{0}, \theta_{2 l}\right)$ in the $(X, Y, \theta)$ system of coordinates, $1 \leq l \leq m$, when $\delta$ tends toward 0 .

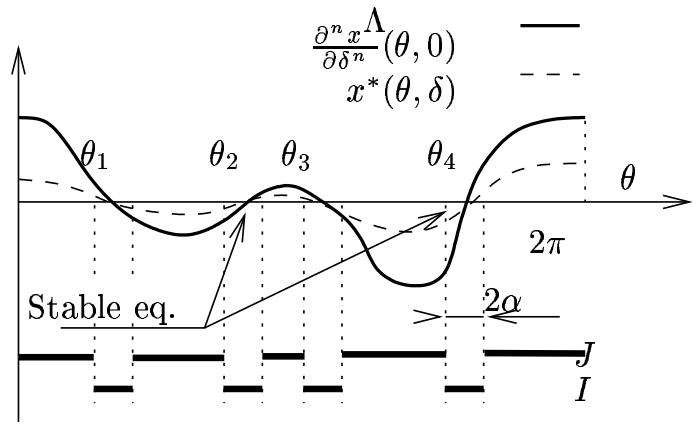

Figure 5: The first non-uniformly zero partial derivative

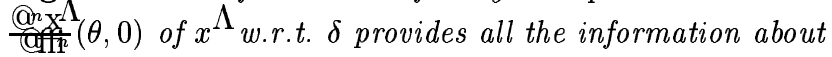
the local minima of Uffifor small values of $\delta$. The values of $\theta$ where this function vanishes with positive slope $\left(\theta_{2}\right.$ and $\theta_{4}$ on this example) are close (by continuity) to the values where $x^{\Lambda}(\theta, \delta)$ vanishes with positive slope for small $\delta$. According to Proposition 6, these values of $\theta$ are stable equilibrium configurations.

Proof: Let us first notice that $\frac{\partial^{n+1}{ }^{1} \Lambda}{\partial \theta \partial \delta^{n}}\left(\theta_{p}, 0\right)$ represents the slope at $\theta_{p}$ of $\frac{\partial^{n} x^{\Lambda}}{\partial \delta^{n}}(\theta, 0)$ seen as a function of $\theta$. Therefore condition (iii) simply means that $\frac{\partial^{n} x^{\Lambda}}{\partial \delta^{n}}(\theta, 0)$ changes sign at each $\theta_{p}$ for $1 \leq p \leq 2 m$.

All the partial derivatives of $x^{*}$ are continuous. Thus, from condition (iii), there exist two positive numbers $\alpha$ and $\delta_{1}$ such that for any positive real number $\delta<\delta_{1}$ and any integer $l$ between 1 and $m$,

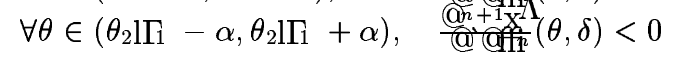

Let us denote by $I$ the union of the intervals of $\theta$ defined above:

$$
I=\bigcup_{1 \leq p \leq 2 m}\left(\theta_{p}-\alpha, \theta_{p}+\alpha\right)
$$

and by $J=\mathbf{S}^{1} \backslash I$ the complement of $I$ as shown on Figure 5 .

The proof consists of two parts:

1. We first prove that over each inter $\chi_{\text {al }}$ constituting $I$, for small fixed $\delta$, the slope $\frac{\partial x}{\partial \theta}$ of $x^{*}$ keeps a 
constant sign and therefore $x^{*}$ vanishes only once over each of these intervals.

2. Then, we show that, for small $\delta, x^{*}$ does not vanish over $J$.

1. Differentiating the equation defined in (i) w.r.t. $\theta$, we get that, for any $k \leq n-1$,

$$
\frac{\partial^{k+1} x^{*}}{\partial \theta \partial \delta^{k}}(\theta, 0)=0
$$

uniformly over $\mathbf{S}^{1}$. If we take a fixed $\theta$ in the interval $\left(\theta_{2 l}-\alpha, \theta_{2 l}+\alpha\right)$, and we consider $\frac{\partial x}{\partial \theta}(\theta, \delta)$ as a function of $\delta$ that we temporarily denote by $f(\delta)$, the above equality can be rewritten

$$
\frac{\partial^{k} f}{\partial \delta^{k}}(0)=0 \quad \text { for } \quad 0 \leq k \leq n-1
$$

Moreover, (15) implies that $\forall \delta \in\left[0, \delta_{1}\right]$,

$$
\frac{\partial^{n} f}{\partial \delta^{n}}(\delta)>0
$$

Therefore, using Taylor-Lagrange formula, for any $\delta \in$ $\left[0, \delta_{1}\right]$, there exists $\beta, 0 \leq \beta \leq 1$ such that

$$
\begin{aligned}
f(\delta) & =\sum_{k=0}^{n-1} \frac{\partial^{k} f}{\partial \delta^{k}}(0) \frac{\delta^{k}}{k !}+\frac{\partial^{n} f}{\partial \delta^{n}}(\beta \delta) \frac{\delta^{n}}{n !} \\
& =\frac{\partial^{n} f}{\partial \delta^{n}}(\beta \delta) \frac{\delta^{n}}{n !}>0
\end{aligned}
$$

This establishes that for any $\delta<\delta_{1}$ and any $\theta \in$ $\left(\theta_{2 l}-\alpha, \theta_{2 l}+\alpha\right), 1 \leq l \leq m, \frac{\partial x}{\partial \theta}(\theta, \delta)>0$. Thus for a fixed $\delta \in\left[0, \delta_{1}\right]$, the function $x^{*}(\theta, \delta)$ of $\theta$ is increasing over $\left(\theta_{2 l}-\alpha, \theta_{2 l}+\alpha\right)$ and cannot vanish more than once over this interval. Using the same reasoning, we can establish that $x^{*}(\theta, \delta)$ is decreasing over the intervals $\left(\theta_{2 l-1}-\alpha, \theta_{2 l-1}+\alpha\right)$ and cannot vanish more than once in each of them either.

2. From condition (ii), when $\theta$ remains in $J, \frac{\partial^{n} x^{\Lambda}}{\partial \delta^{n}}(\theta, 0)$ does not vanish. As $J$ is compact, $\left|\frac{\partial^{n} x}{\partial \delta^{n}}(\theta, 0)\right|$ admits a positive lower bound over $J$, that we denote by $M$

$$
M=\min \left\{\left|\frac{\partial^{n} x^{*}}{\partial \delta^{n}}(\theta, 0)\right|, \theta \in J\right\}>0
$$

¿From the uniform continuity of $\frac{\partial^{n} x^{\Lambda}}{\partial \delta^{n}}(\theta, \delta)$ over the compact set $J \times\left[0, \delta_{1}\right]$, there exists $\delta_{2}, 0<\delta_{2} \leq \delta_{1}$ such that for any $\delta \in\left[0, \delta_{2}\right]$ and any $\theta \in J$,

$$
\left|\frac{\partial^{n} x^{*}}{\partial \delta^{n}}(\theta, \delta)-\frac{\partial^{n} x^{*}}{\partial \delta^{n}}(\theta, 0)\right|<M
$$

and using (20),

$$
\left|\frac{\partial^{n} x^{*}}{\partial \delta^{n}}(\theta, \delta)\right|>0
$$

We can apply again Taylor-Lagrange relation. To keep the same notation, $f(\delta)$ denotes now $x^{*}(\theta, \delta)$ considered as a function of $\delta$ for a fixed value of $\theta \in J$. We get from condition (i) that condition (16) is satisfied and from (21) that $\frac{\partial^{n} f}{\partial \delta^{n}}(\delta)$ does not vanish over $\left[0, \delta_{2}\right]$ and thus keeps a constant sign over this interval. Condition (17) (or its counterpart $\frac{\partial^{n} f}{\partial \delta^{n}}(\delta)<0$ ) is thus satisfied over $\left[0, \delta_{2}\right]$. We can reuse (18) and (19) (replacing $>$ by $<$ if $\left.\frac{\partial^{n} f}{\partial \delta^{n}}(\delta)<0\right)$ to conclude that $x^{*}(\theta, \delta)$ does not vanish over $\left[0, \delta_{2}\right]$ and has the same sign as $\frac{\partial^{n} x}{\partial \delta^{n}}(\theta, 0)$.

If we now consider a fixed $\delta<\delta_{2}$ and if we recall that $\frac{\partial^{n} x}{\partial \delta^{n}}(\theta, 0)$ changes sign between two subintervals constituting $J$, we can conclude that $x^{*}(\theta, \delta)$ vanishes exactly once over each $\left(\theta_{l}-\alpha, \theta_{l}+\alpha\right), 1 \leq$ $l \leq 2 m$ at a value of $\theta$ that we denote by $\tilde{\theta}_{l}(\delta)$. The stable equilibrium configurations of the part are expressed in the $(X, Y, \theta)$ system of coordinates by $\left(X^{*}\left(\tilde{\theta}_{2 l}(\delta), \delta\right), Y^{*}\left(\tilde{\theta}_{2 l}(\delta), \delta\right), \tilde{\theta}_{2 l}\right)$. As $\alpha$ can be chosen as small as desired by making $\delta$ tend toward $0, \lim _{\delta \rightarrow 0} \tilde{\theta}_{2 l}(\delta)=\theta_{2 l} . \quad X^{*}, Y^{*}$ being continuous, the stable equilibrium configurations converge toward $\left(X_{0}, Y_{0}, \theta_{2 l}\right)$ as $\delta$ tends toward 0.

Let us point out that in most cases, the first non uniformly zero partial derivative $\frac{\partial^{n} x}{\partial \delta^{n}}(\theta, 0)$ has simple roots (we will see later that these partial derivatives are trigonometric polynomials in $\theta$ ) and Theorem 7 can be used. This theorem extends results from [12]. In the next section we use Theorem 7 to compute all the equilibrium configurations of a part under the radialconstant force field. 


\section{An algorithm to Compute all Equi- librium Configurations of a Part}

This section is organized as follows. First we explain how to iteratively compute expressions of the partial derivatives $\frac{\partial^{k} x}{\partial \delta^{k}}(\theta, 0)$ w.r.t. the partial derivatives of the lifted radial potential field $V$ evaluated at $(0,0)$ : $\frac{\partial^{n} V}{\partial X^{k} \partial Y^{n} \Gamma_{k}}(0,0)$. These expressions enable us to compute the equilibrium configurations of a part using Theorem 7 . We detail all the necessary operations in an algorithm. Then we make some comments about the computation of $\frac{\partial^{n} V}{\partial X^{k} \partial Y^{n} \mathbb{T}_{k}}(0,0)$. Finally, an example illustrates the algorithm.

Expressions of $\frac{\partial^{k} x}{\partial \delta^{k}}(\theta, 0)$

Differentiating Equation (13) yields

$$
\frac{\partial^{k} x^{*}}{\partial \delta^{k}}(\theta, \delta)=\cos \theta \frac{\partial^{k} X^{*}}{\partial \delta^{k}}(\theta, \delta)-\sin \theta \frac{\partial^{k} Y^{*}}{\partial \delta^{k}}(\theta, \delta)
$$

To compute an expression of $\frac{\partial^{k} x}{\delta^{k}}(\theta, 0)$ we need thus to compute expressions of $\frac{\partial^{k} X^{k}}{\partial \delta^{k}}(\theta, 0)$ and $\frac{\partial^{k} Y}{\partial \delta^{k}}(\theta, 0)$.

By definition, $\left(X^{*}, Y^{*}\right)$ minimizes $U_{\theta, \delta}$. Thus the partial derivatives $\frac{\partial U_{\theta, \delta}}{\partial X}$ and $\frac{\partial U_{\theta, \delta}}{\partial Y}$ vanish at $\left(X^{*}, Y^{*}\right)$. Using expression (12), this statement is equivalent to

$$
\begin{aligned}
& \frac{\partial V}{\partial X}\left(X^{*}(\theta, \delta), Y^{*}(\theta, \delta)\right)+\delta|S| \sin \theta=0 \\
& \frac{\partial V}{\partial Y}\left(X^{*}(\theta, \delta), Y^{*}(\theta, \delta)\right)+\delta|S| \cos \theta=0
\end{aligned}
$$

By successively differentiating this system w.r.t. $\delta$ and by evaluating the resulting system at $\delta=0$ we will get expressions of $\frac{\partial^{k} X}{\partial \delta^{k}}(\theta, 0)$ and $\frac{\partial^{k} Y \Lambda}{\partial \delta^{k}}(\theta, 0)$. We show below the first step of this process by computing an expression of $\frac{\partial x}{\partial \delta}(\theta, 0)$. Then we will address the general case with higher order $\frac{\partial^{k} x^{\Lambda}}{\partial \delta^{k}}(\theta, 0)$.

Distinct Pivot Point and Center of Mass If $\left(X_{0}, Y_{0}\right) \neq(0,0)$, we express this vector in polar coordinates: $\left(X_{0}, Y_{0}\right)=(\rho \cos \psi, \rho \sin \psi), \rho>0$. Then, from (10),

$$
x^{*}(\theta, 0)=\rho \cos (\theta+\psi) .
$$

$x^{*}(\theta, 0)$ vanishes with positive slope for $\theta=\frac{3 \pi}{2}-\psi$ and Theorem 7 (with $n=0$ ) permits to conclude that when $\delta$ tends toward 0 , the stable equilibrium configuration is unique and tends toward $\left(X_{0}, Y_{0}, \frac{3 \pi}{2}-\psi\right)$ in the new system of coordinates, that is $\left(-\rho, 0, \frac{3 \pi}{2}-\psi\right)$ in the standard system of coordinates.

Same Pivot Point and Center of Mass We assume now that $\left(X_{0}, Y_{0}\right)=(0,0)$. Let us differentiate once the above system w.r.t. $\delta$. We get

$$
\begin{aligned}
& \frac{\partial^{2} V}{\partial X^{2}} \frac{\partial X^{*}}{\partial \delta}+\frac{\partial^{2} V}{\partial X \partial Y} \frac{\partial Y^{*}}{\partial \delta}+|S| \sin \theta=0 \\
& \frac{\partial^{2} V}{\partial X \partial Y} \frac{\partial X^{*}}{\partial \delta}+\frac{\partial^{2} V}{\partial Y^{2}} \frac{\partial Y^{*}}{\partial \delta}+|S| \cos \theta=0
\end{aligned}
$$

where the partial derivatives of $V$ are evaluated at $\left(X^{*}(\theta, \delta), Y^{*}(\theta, \delta)\right)$ and $\frac{\partial X}{\partial \delta}, \frac{\partial Y}{\partial \delta}$ are evaluated at $(\theta, \delta)$. If we take $\delta=0$ in this system, we get

$$
\text { Hess } V(0,0)\left(\begin{array}{c}
\frac{\partial X}{\partial \delta \Lambda}(\theta, 0) \\
\frac{\partial Y}{\partial \delta}(\theta, 0)
\end{array}\right)=-|S|\left(\begin{array}{c}
\sin \theta \\
\cos \theta
\end{array}\right) \text {. }
$$

According to Proposition 3, the above Hessian is invertible, so that expressions of $\frac{\partial X}{\partial \delta}(\theta, 0)$ and $\frac{\partial Y}{\partial \delta}(\theta, 0)$ and thus of $\frac{\partial x}{\partial \delta}(\theta, 0)$ are obtained by inversion of system (24). Let us notice that these expressions are trigonometric polynomials of $\theta$.

To get higher order derivatives of $X^{*}$ and $Y^{*}$, we need to differentiate several times (24). Let us point out a property of this system.

Proposition 8 Differentiating $k-1$ times system (2223) ( $k \geq 2)$ yields a system of the form:

$$
\text { Hess } V\left(X^{*}(\theta, \delta), Y^{*}(\theta, \delta)\right)\left(\begin{array}{c}
\frac{\partial^{k} X}{\partial \delta^{k} \Lambda}(\theta, \delta) \\
\frac{\partial^{k} Y}{\partial \delta^{k}}(\theta, \delta)
\end{array}\right)=E_{k}
$$

where $E_{k}$ is a polynomial expression of variables $\frac{\partial^{l} X^{\Lambda}}{\partial \delta^{l}}(\theta, \delta), \quad \frac{\partial^{l} Y^{\Lambda}}{\partial \delta^{l}}(\theta, \delta)$ for $1 \leq l \leq k-1$. The coefficients of this polynomial are functions of the $\frac{\partial^{i} V}{\partial X^{j} \partial Y^{i} \mathrm{I}_{j}}\left(X^{*}(\theta, \delta), Y^{*}(\theta, \delta)\right)$, for $2 \leq i \leq k+1$ and $0 \leq j \leq i$.

Proof: This proposition can be easily proved by induction. For $k=2$, if we differentiate once (22-23), we find that the proposition is satisfied with

$$
E_{2}=-\left(\begin{array}{l}
V_{3,0} X_{1}^{* 2}+2 V_{2,1} X_{1}^{*} Y_{1}^{*}+V_{1,2} Y_{1}^{* 2} \\
V_{2,1} X_{1}^{* 2}+2 V_{1,2} X_{1}^{*} Y_{1}^{*}+V_{0,3} Y_{1}^{* 2}
\end{array}\right)
$$

where $V_{i, j}=\frac{\partial^{i+j} V}{\partial X^{i} \partial Y^{j}}\left(X^{*}(\theta, \delta), Y^{*}(\theta, \delta)\right), \quad X_{1}^{*}=$ $\frac{\partial X}{\partial \delta}(\theta, \delta), Y_{1}^{*}=\frac{\partial Y^{\prime}}{\partial \delta}(\theta, \delta)$. 
Now, we assume that the proposition is satisfied at order $k$, that is $E_{k}$ in (25) is of the correct form. Let us differentiate again this equality w.r.t. $\delta$. The left hand side becomes

$$
\begin{aligned}
& \text { Hess } V\left(X^{*}(\theta, \delta), Y^{*}(\theta, \delta)\right)\left(\begin{array}{c}
\frac{\partial^{k+1} X}{\partial \delta^{k+1}}(\theta, \delta) \\
\frac{\partial^{k+1} Y}{\partial \delta^{k+1}}(\theta, \delta)
\end{array}\right)+ \\
& \left(\begin{array}{cc}
V_{3,0} X_{1}^{*}+V_{2,1} Y_{1}^{*} & V_{2,1} X_{1}^{*}+V_{1,2} Y_{1}^{*} \\
V_{2,1} X_{1}^{*}+V_{1,2} Y_{1}^{*} & V_{1,2} X_{1}^{*}+V_{0,3} Y_{1}^{*}
\end{array}\right)\left(\begin{array}{c}
X_{k}^{*} \\
Y_{k}^{*}
\end{array}\right)
\end{aligned}
$$

Notice that the first term of this sum is exactly the left hand side of (25) at order $k+1$. The second term can be included in $E_{k+1}$ : it is of the correct form.

It remains to check that if $E_{k}$ is of the form described in the proposition, so is $E_{k+1}$. This test is straightforward.

The expressions involved in the above proposition are rather complex. That is why we only give their form instead of writing them here. However it is important to notice the structure of the successive relations expressed by (25). If we take $\delta=0$ in these equations, we get linear systems in $\left(\frac{\partial^{k} X}{\partial \delta^{k}}(\theta, 0), \frac{\partial^{k} Y^{\Lambda}}{\partial \delta^{k}}(\theta, 0)\right)$. These systems are invertible since Hess $V$ is positive definite. By inverting these systems iteratively, we get successive expressions of $\frac{\partial^{k} X^{\Lambda}}{\partial \delta^{k}}(\theta, 0)$ and $\frac{\partial^{k} Y}{\partial \delta^{k}}(\theta, 0)$ and thus of $\frac{\partial^{k} x^{\Lambda}}{\partial \delta^{k}}(\theta, 0)$ w.r.t. the partial derivatives of $V$ at $(0,0)$. It can be verified that these expressions are trigonometric polynomials in $\theta$. This iterative procedure is the core of the algorithm presented below.

\section{The Algorithm}

Using the above developments, the roots of the first non-uniformly zero $\frac{\partial^{k} x}{\partial \delta^{k}}(\theta, 0)$ can be computed by the algorithm presented in Table 1 . This algorithm needs the partial derivatives of $V$ evaluated at $(0,0)$ and a function, minimize, that computes the unique minimum of $V$.

\section{The Partial Derivatives of $V$}

If the part is polygonal, we can express exactly the partial derivatives of $V$ and thus evaluate them for any $(X, Y)$. In this case, minimize can numerically minimize $V$ using a gradient method and return an approximation of $\left(X_{0}, Y_{0}\right)$.

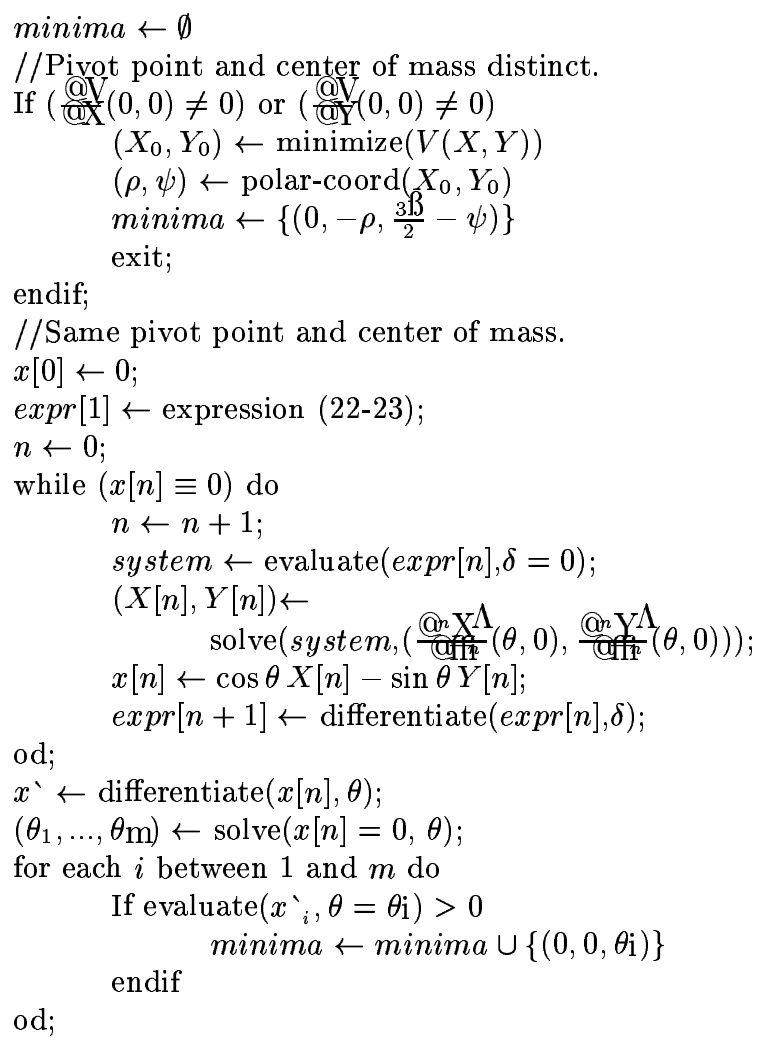

Table 1: Algorithm that computes the local minima of the lifted radial-constant field Uffifor small $\delta$.

If the part is not a poligon, the partial derivatives of $V$ can be computed numerically. We do not give details about these numerical computations. Some discussion can be found in [11].

\section{Example}

Let us compute the stable equilibrium configurations of the part represented in Figure 6. For this polygonal part, symmetric by rotation of angle $\frac{\pi}{2}$, we have computed using Maple the expressions of $\frac{\partial V}{\partial X}$ and $\frac{\partial V}{\partial X}$. These expressions are very long, we cannot report them here. Differentiating symbolically these expressions, we obtained higher order derivatives of $V$ that we evaluated at $(0,0)$ :

$$
\begin{aligned}
& \frac{\partial^{2} V}{\partial X^{2}}(0,0)=\frac{\partial^{2} V}{\partial Y^{2}}(0,0)= \\
& \frac{8 \sqrt{10}}{5}\left(\operatorname{Argsinh} 3-\operatorname{Argsinh} \frac{1}{2}\right)
\end{aligned}
$$




$$
\begin{aligned}
& \frac{\partial^{2} V}{\partial X \partial Y}(0,0)=0 \\
& \frac{\partial^{3} V}{\partial X^{3}}(0,0)=\frac{\partial^{3} V}{\partial X^{2} \partial Y}(0,0)=0 \\
& \frac{\partial^{3} V}{\partial X \partial Y^{2}}(0,0)=\frac{\partial^{3} V}{\partial Y^{3}}(0,0)=0 \\
& \frac{\partial^{4} V}{\partial X^{4}}(0,0)=\frac{\partial^{4} V}{\partial Y^{4}}(0,0)=\frac{\sqrt{2}}{2}-3 \\
& \frac{\partial^{4} V}{\partial X^{3} \partial Y}(0,0)=\frac{\partial^{4} V}{\partial X \partial Y^{3}}(0,0)=0 \\
& \frac{\partial^{4} V}{\partial X^{2} \partial Y^{2}}(0,0)=\frac{\sqrt{2}}{2}
\end{aligned}
$$

We apply now the successive steps of the algorithm described in Table 1 to the part.

To simplify the notation, let us write $\lambda=$ $\frac{\partial^{2} V}{\partial X^{2}}(0,0)=\frac{\partial^{2} V}{\partial Y^{2}}(0,0)$. Substituting $\delta=0$ in $(22-23)$ yields

$$
\begin{aligned}
& \lambda \frac{\partial X^{*}}{\partial \delta}(\theta, 0)+|S| \sin \theta=0 \\
& \lambda \frac{\partial Y^{*}}{\partial \delta}(\theta, 0)+|S| \cos \theta=0
\end{aligned}
$$

Inverting this system yields

$$
\frac{\partial X^{*}}{\partial \delta}(\theta, 0)=-\frac{|S|}{\lambda} \sin \theta \quad \frac{\partial Y^{*}}{\partial \delta}(\theta, 0)=-\frac{|S|}{\lambda} \cos \theta
$$

Substituting these equalities in (13) gives as expected

$$
x^{*}(\theta, 0)=0 .
$$

Now, differentiating again (22-23) and substituting $\delta=$ 0 , we get

$$
\begin{aligned}
& \lambda \frac{\partial^{2} X^{*}}{\partial \delta^{2}}+V_{3,0} X_{1}^{* 2}+2 V_{2,1} X_{1}^{*} Y_{1}^{*}+V_{1,2} Y_{1}^{* 2}=0 \\
& \lambda \frac{\partial^{2} Y^{*}}{\partial \delta^{2}}+V_{2,1} X_{1}^{* 2}+2 V_{1,2} X_{1}^{*} Y_{1}^{*}+V_{0,3} Y_{1}^{* 2}=0
\end{aligned}
$$

where $V_{i, j}=\frac{\partial^{i+j} V}{\partial X^{i} \not Y^{j}}(0,0), X_{1}^{*}=\frac{\partial X}{\partial \delta}(\theta, 0), Y_{1}^{*}=$ $\frac{\partial Y}{\partial \delta}(\theta, 0)$ and $\frac{\partial^{2} X}{\partial \delta^{2}}$ and $\frac{\partial^{2} Y}{\partial \delta^{2}}$ are evaluated at $(\theta, 0)$. From this system, we extract expressions of the second order partial derivatives of $X^{*}$ and $Y^{*}$ :

$$
\frac{\partial^{2} X^{*}}{\partial \delta^{2}}(\theta, 0)=0 \quad \frac{\partial^{2} Y^{*}}{\partial \delta^{2}}(\theta, 0)=0
$$

Thus

$$
\frac{\partial^{2} x^{*}}{\partial \delta^{2}}(\theta, 0)
$$

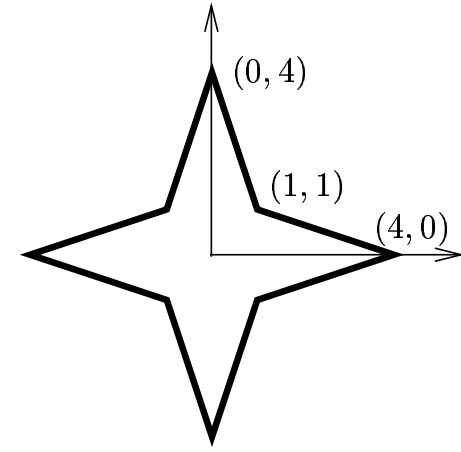

Figure 6: Polygonal part symmetric by rotation of angle $\frac{\beta}{2}$.

To get the third order partial derivatives of $X^{*}$ and $Y^{*}$, we need to differentiate twice (22-23) w.r.t. $\delta$ and take $\delta=0$. This yields two equations of the form:

$$
\begin{aligned}
& \lambda \frac{\partial^{3} X^{*}}{\partial \delta^{3}}(\theta, 0)+\ldots=0 \\
& \lambda \frac{\partial^{3} Y^{*}}{\partial \delta^{3}}(\theta, 0)+\ldots=0
\end{aligned}
$$

where $\cdots$ stands for already evaluated expressions. From this system, we get

$$
\begin{aligned}
& \frac{\partial^{3} X^{*}}{\partial \delta^{3}}(\theta, 0)=2048 \frac{\sin \theta\left((2 \sqrt{2}+6) \cos ^{2} \theta+\sqrt{2}-6\right)}{\lambda^{4}} \\
& \frac{\partial^{3} Y^{*}}{\partial \delta^{3}}(\theta, 0)=-2048 \frac{\cos \theta\left((2 \sqrt{2}+6) \cos ^{2} \theta-3 \sqrt{2}\right)}{\lambda^{4}}
\end{aligned}
$$

and finally

$$
\frac{\partial^{3} x^{*}}{\partial \delta^{3}}(\theta, 0)=\frac{1024 \sqrt{2}+3072}{\lambda^{4}} \sin 4 \theta .
$$

We recall that $\lambda=\frac{8 \sqrt{10}}{5}\left(\operatorname{Argsinh} 3-\operatorname{Argsinh} \frac{1}{2}\right)>0$.

$x^{*}$ sqtisfies the hypotheses of Theorem 7 for $n=$ 3. $\frac{\partial^{3} x \Lambda}{\partial \delta^{3}}(\theta, 0)$ vanishes 8 times, 4 times with negative slope and 4 times with positive slope (for $\theta=i \frac{\pi}{2}$, $i=0,1,2,3)$. We conclude that for small values of $\delta$, the part has 4 stable equilibrium positions converging toward $\left(0,0, i \frac{\pi}{2}\right)$ when $\delta$ tends toward 0 . The part is thus positioned up to part symmetry by the force field.

\section{Conclusion}

We have proposed a method to compute all stable equilibrium configurations of a part subjected to the com- 
bination of a unit radial force field with a small constant field. This method is general and can be applied to any part, symmetric or not. Beyond this method, this paper reports a comprehensive study of the action of radial and small constant potential fields over parts. It proposes an interesting characterization of local minima of the lifted potential field using the equilibrium curve and its partial derivatives for $\delta=0$. Some questions remain open however. All these results are asymptotic for small $\delta$. The general case with any $\delta$ between 0 and 1 is far more difficult since we do not have any expression of the equilibrium curve in general and the stable equilibrium configurations are the roots of the equilibrium curve. For this case, numerical methods will probably be necessary. The rate of convergence under the fields described in this paper, as well as under previously proposed fields, is an open question.

Acknowledgments This work was performed while Florent Lamiraux was with the Department of Computer Science at Rice University. The authors are grateful to Karl Böringer and Bruce Donald for many discussions. Work on this paper by Lydia Kavraki and Florent Lamiraux was partially supported by NSF IRI-970228 and NSF CISE SA1728-21122N.

\section{References}

[1] S. Akella, W. Huang, K. Lynch, and M. Mason. Planar manipulation on a conveyor with a one joint robot. In G. Giralt and G. Hirzinger, editors, International Symposium on Robotics Research, pages 265-276. Springer, London, 1996.

[2] D. Biegelsen, W. Jackson, A. Berlin, and P. Cheung. Air jet arrays for precision positional control of flexible media. In Int. Conf. on Micromechatronics for Information and Precision Equipment, pages 631-634, Tokyo, Japan, 1997.

[3] K. Böhringer, V. Bhatt, and K. Goldberg. Sensorless manipulation using transverse vibrations of a plate. In IEEE Int. Conf. on Rob. and Autom., pages 19891996, 1995.

[4] K. Böhringer, B. Donald, and N. MacDonald. Programmable vector fields for distributed manipulation, with application to mems actuator arrays and vibratory part feeders. International Journal on Robotics Research, 18:168-200, Feb. 1999.
[5] K.-F. Böhringer, B. R. Donald, L. E. Kavraki, and F. Lamiraux. Part orientation with one or two stable equilibria using programmable vector fields. to appear in the IEEE Transactions on Robotics and Automation.

[6] K.-F. Böhringer, B. R. Donald, and N. C. MacDonald. Upper and lower bounds for programmable vector fields with applications to MEMS and vibratory plate parts feeders. In J. Laumond and M. Overmars, editors, Algorithms for Robotic Motion and Manipulation, pages 255-276, Natick, MA, 1997. AK Peters.

[7] J. Canny and K. Y. Goldberg. Risc for industrial robotics: recent results and open problems. In $I E E E$ Int. Conf. on Rob. and Autom., pages 1951-1958, 1994.

[8] M. Erdmann and M. Mason. An exploration of sensorless manipulation. IEEE Tr. on Rob. and Autom., 4(4):369-379, 1988.

[9] K. Y. Goldberg. Orienting polygonal parts without sensors. Algorithmica, 10:201-225, 1993.

[10] L. E. Kavraki. Part orientation with programmable vector fields: Two stable equilibria for most parts. In IEEE Int. Conf. on Robotics and Automation, pages 2446-2451, 1997.

[11] F. Lamiraux and L. Kavraki. Part positioning using radial and constant fields: Modeling and computation of all equilibrium configurations. in preparation.

[12] F. Lamiraux and L. E. Kavraki. Positioning symmetric parts using a combination of a unit-radial and a constant force fields. to appear in IEEE International conference on Robotics and Automation, San Francisco, 2000 .

[13] W. Liu and P. Will. Part manipulation on an intelligent motion surface. In International Conference on Intelligent Robots and Systems, pages 399-404, Pittsburg, PA, Aug. 1995. IEEE/RSJ.

[14] J. Luntz, W. Messner, and H. Choset. Velocity field design for parcel manipulation on the virtual vehicule, a discrete distributed actuator array. In P. Agarwal, L. E. Kavraki, and M. Mason, editors, Robotics: The Algorithmic Perspective, pages 35-47. AK Peters, Natick, MA, 1998.

[15] D. S. Reznik and J. Canny. Universal part manipulation in the plane with a single horizontally-vibrating plate. In P. K. Agarwal, L. E. Kavraki, and M. Mason, editors, Robotics: The Algorithmic Perspective, pages 21-47, Natick, MA, 1998. AK Peters. 
[16] J. Wiegley, K. Goldberg, M. Peshkin, and M. Brokowski. A complete algorithm for designing passive fences to orient parts. In International Conference on Robotics and Automation, pages 1133-1139, 1996. 